\title{
FKBPL Is a Potential Prognostic Biomarker and Correlated with Immune Infiltrates and T Cells Exhaustion in Hepatocellular Carcinoma
}

\author{
Feng Tian ${ }^{1}$, Lifu Wang ${ }^{1}$, Baiqiang $\mathrm{Ma}^{1}$, Bingzhen $\mathrm{Li}^{1}$, Daxia $\mathrm{Ca}^{2}{ }^{2}$ * \\ ${ }^{1}$ Department of General surgery, Lishui People's Hospital, the Six Affiliated Hospital of Wenzhou Medical University, Lishui, China \\ ${ }^{2}$ Institute of Hematology, Jinan University, Guangzhou, P. R. China
}

Email address:

tfcdx620@stu2018.jnu.edu.cn (Daxia Cai)

${ }^{*}$ Corresponding author

To cite this article:

Feng Tian, Lifu Wang, Baiqiang Ma, Bingzhen Li, Daxia Cai. FKBPL Is a Potential Prognostic Biomarker and Correlated with Immune Infiltrates and T Cells Exhaustion in Hepatocellular Carcinoma. Cancer Research Journal. Vol. 8, No. 4, 2020, pp. 82-93. doi: $10.11648 /$ j.crj.20200804.14

Received: November 11, 2020; Accepted: November 20, 2020; Published: November 27, 2020

\begin{abstract}
Hepatocellular carcinoma (HCC) is a malignant tumor with high mortality and poor prognosis. And FKBPL plays a crucial role on the development of cancers. This study aims to explore the prognostic potential of FKBPL and comprehensively analyze the correlations between FKBPL, immune infiltration, and T cells function in HCC. GEO database, Oncomine databases, TIMER2.0 and GEPIA2 were used to analyze FKBPL expression, relationship between FKBPL expression and survival of HCC patients as well as immune infiltration, related gene marker sets and T cells exhaustion in HCC. We used STRING database to analysis PPI (protein-protein interaction) for FKBPL and visualization by Cytoscape. Metascape was used for enrichment analysis. FKBPL upregulates in most of the cancer types including HCC. Survival analysis demonstrated that FKBPL shows significant impact on survival of HCC patients with overall survival and disease-free survival. And survival rate of HCC patients with high FKBPL expression was significantly correlated with race, gender and tumor purity of HCC patients. Moreover, FKBPL expression positively correlates with high immune infiltration levels in most of the immune cells and functional $\mathrm{T}$ cells including exhausted T cells, Th1, Th2 and Tfh. Specifically, multiple key genes of exhausted T cells comprising PD-1, CTLA4, LAG3, TIM-3, and GZMB have remarkable interaction with FKBPL. At last, we found10 genes including PRRT1, ANKRD49, RBCK1, CSNK1D, HSP90AA1, HSP90AB1, GTSE1, CDKN1A, AVPR2, and EGFL8 were interacted with FKBPL. Enrichment analysis showed that apoptotic signaling pathway, regulation of myeloid cell differentiation, cell death in response to oxidative stress, PIP3 activates AKT signaling may involve in HCC tumor progression about FKBPL. In conclusion, FKBPL is a potential prognostic biomarker and correlated with immune infiltrates and T cells exhaustion in HCC.
\end{abstract}

Keywords: Hepatocellular Carcinoma (HCC), FKBPL, Prognosis, Immunotherapy, Immune Infiltration, T Cells Exhaustion

\section{Introduction}

Hepatocellular carcinoma (HCC) is ranked as the sixth most common neoplasm and the third leading cause of cancers death, and metastasis plays an important role in the poor prognosis of HCC patients [1]. Despite clinical comprehensive therapy comprising liver transplantation, surgical resection, chemotherapy, radiotherapy, interventional therapy and immunotherapy have improved clinical outcome in HCC patients. Most HCC patients have difficulty in early detection and effective treatment, and the postoperative recurrence rate is high, leading to poor clinical prognosis. According to statistics, the 3 years relative survival in HCC patients improved from $19.8 \%$ in $1998-2002,22.4 \%$ in $2003-2007,30.6 \%$ in $2008-2012$ up to $31.0 \%$ in $2013-2016$, those are still dismal numbers [2]. Previous studies have shown that immune infiltration and tumor microenvironment play an important role in the progression of $\mathrm{HCC}$, such as programmed death-1 (PD-1), cytotoxic $\mathrm{T}$ lymphocyte antigen-4 (CTLA-4), regulatory $\mathrm{T}$ cell (Treg) infiltration, natural killer (NK) cells, CD8+ T cells, helper T (Th)-17, tumor-associated macrophages (TAMs), STAT3, and M1/M2 Macrophage[3]. Moreover, a growing number of studies have 
found that the tumor-infiltrating lymphocytes, such as tumor-associated macrophages (TAMs) and neoplastic infiltrates neutrophils (TINs) [4], hematopoietic stem cell [5, 6], myeloid derived suppressor cells (MDSC) [7, 8], T cell follicular helper [9], endothelial cell [10], and mast cell [11] can affect the prognosis and efficacy of patients chemotherapy and immunotherapy. In addition, effective immunotherapy derived from several biomarkers, such as immunotherapy with checkpoint inhibitors and chimeric antigen receptor (CAR) T-cell therapies, particularly inhibitors of the anti-programed cell death 1 (PD-1) and anti-programed cell death 1 ligand 1 (PD-L1) axis, have been thought better therapeutic effects on HCC, and combination of checkpoint blockade inhibitors and other methods may enhance the efficacy in $\mathrm{HCC}[3,12]$. However, more novel and effective biomarkers for the prediction or enhancement of immunotherapy response need to be explored.

FKBPL (FKBP Prolyl Isomerase Like), a endogenous protein angiogenesis inhibitor [13], has been proposed as a novel prognostic and predictive biomarker in cancer, as well as therapeutic target for breast cancer patients' diagnosis and treatment [14]. Recently, it has been confirmed that FKBPL-CD44 pathway can be thought as the diagnostic and therapeutic potential of the angiogenesis markers in preeclampsia [15]. However, the correlations between FKBPL expression, prognosis, and immune infiltration in $\mathrm{HCC}$ remain unclear.

In this study, we comprehensively analyzed FKBPL expression and its prognostic value in HCC using multiple databases including Tumor Immune Estimation Resource2.0 (TIMER2.0), Gene Expression Profiling Interactive Analysis 2 (GEPIA2), GEO database, Oncomine database. The relationships between FKBPL expression and immune infiltration in HCC were investigated via TIMER2.0 database and GEPIA2 database. We found that FKBPL is a potential prognostic biomarker and correlated with immune infiltrates in HCC and provided novel direction to understand the interactions between FKBPL expression, tumor infiltration, and $\mathrm{T}$ cells exhaustion. At last, protein-protein interactions was predicted by STRING database and enrichment analysis was performed through Metascape database.

\section{Materials and Methods}

\subsection{Data Mining and Related Data Analysis Validation Databases}

GEPIA2(Gene Expression Profiling Interactive Analysis 2) database[16] (http://gepia2.cancer-pku.cn/\#index) is an online database for cancer and normal gene expression and interactive analysis based on the Cancer Genome Atlas (TCGA) and GTEx (Genotype-Tissue Expression) data. For our liver, it includes 369 liver hepatocellular carcinoma and 50 paired adjacent or non-tumor liver tissues from the TCGA and the liver GTEx projects. It can provide differential expression analysis, profiling plotting, correlation analysis, patient survival analysis and similar gene detection. In our study, the correlations between FKBPL and gene markers of tumor-infiltration immune cells were also performed using Spearman's correlation coefficient in "correlation analysis.". Differentially expressed FKBPL was analyzed in "General" and "Expression DIY". The relationship between the expression of FKBPL and the survival curves of HCC patients including OS and DFS was analyzed in "Survival Analysis".

HCC and adjacent normal tissue gene expression profiles of GSE25097 [17], GSE55092 [18], GSE84598 [19], GSE87630 [20], GSE45436 and GSE45267 [21], GSE76297 and GSE76311 [22], GSE60502 [23], GSE121248 [24], and GSE46408 [25] were downloaded from GEO (http://www.ncbi.nlm.nih.gov/geo/) database.

Oncomine

database (https://www.oncomine.org/resource/login.html) can be used to analyze the expression level of the FKBPL gene in HCC, and the threshold was determined according to the following values: $p$-value $\leq 1 \mathrm{E}-4$, fold change $\geq 2$ and top $10 \%$ gene rank.

TIMER2.0 database [26] (http://timer.cistrome.org/) is a comprehensive resource for systematical analysis of immune infiltrates across diverse cancer types. To explore tumor immunological, clinical and genomic features comprehensively, the database providers analyzed gene expression data including 10897 samples across 32 cancer types from The Cancer Genome Atlas (TCGA) to estimate the abundance of six tumor-infiltrating immune cells (TIICs) subsets(including B cells, CD4+ T cells, CD8+ T cells, macrophages, neutrophils, and dendritic cells), fibroblast, hematopoietic stem cell, common lymphoid progenitor, myeloid derived suppressor cells (MDSC), granulocyte-monocyte progenitor, $\mathrm{T}$ cell follicular helper, endothelial cell, $\mathrm{T}$ cell gamma delta, eosinophil, neutrophil, monocyte, and mast cell. In our study, differentially expressed FKBPL across 32 cancer types was analyzed by "Gene-DE module" in "Cancer Exploration Modules". We used "Gene module" in "Immune Association module" to analyze the correlations between FKBPL expression in HCC and the abundance of six TIIC subsets. Statistical significance of differential FKBPL expression was evaluated using Wilcoxon test. The correlations of FKBPL expression with immune infiltration were evaluated by purity-corrected partial Spearman's correlation and statistical significance. Moreover, correlations between FKBPL expression and gene markers of tumor-infiltration immune cells were investigated via "Gene_Corr module" in "Cancer Exploration Modules". In addition, we used "Gene-Outcome module" in "Cancer Exploration Modules" to analyze the clinical relevance of FKBPL gene expression across HCC including gender, race, purity, age and stage.

STRING database [27] (https://string-db.org/) is a database of known and predicted protein-protein interactions. The interactions include direct (physical) and indirect (functional) associations; they stem from computational prediction, from knowledge transfer between organisms, and from interactions aggregated from other (primary) databases. In our study, we used STRING database to reveal the 10 genes that interact with FKBPL for PPI analysising, and then visualized using Cytoscape software. 
Metascape [28] (http://metascape.org) was an online database that integrates over forty bioinformatics knowledge bases enabling the extraction of abundant annotations, as well as the identification of enriched pathways and the construction of protein-protein interaction networks from lists of gene and protein identifiers. Gene Ontology (GO) terms for the biological process (BP), cellular component (CC) and molecular function (MF) categories as well as Kyoto Encyclopedia of Genes and Genomes (KEGG) pathways were enriched. In our study, Metascape was introduced to perform functional and pathway enrichment analysis of the top 500 genes similar to FKBPL in HCC, which were downloaded from the GEPIA2 database.

\subsection{Statistical Analysis}

Differences of gene expression between the individual groups were analyzed using student $t$ test by GraphPad Prism $8,{ }^{* * *} \mathrm{p}<0.001,{ }^{* *} \mathrm{p}<0.01,{ }^{*} \mathrm{p}<0.05$. The other data was downloaded from open database analysis, and see the corresponding database.

\section{Results}

\subsection{An Overview of the Expression Level of FKBPL in Different Types of Human Cancers}

To explore the differential expression of FKBPL in human cancers, GEPIA2 database (Figure 1A) and TIMER database (Figure 1B) were respectively used to analyze the expression of FKBPL in human 32 tumors. This analysis revealed that the FKBPL expression was significantly higher compared to the normal tissues in bladder urothelial carcinoma (BLCA), breast invasive carcinoma (BRCA), colon adenocarcinoma (COAD), kidney renal clear cell carcinoma (KIRC), kidney renal papillary cell carcinoma (KIRP), liver hepatocellular carcinoma (LIHC), lung adenocarcinoma (LUAD), lung squamous cell carcinoma (LUSC), Prostate adenocarcinoma (PRAD), skin cutaneous melanoma (SKCM), and uterine corpus endometrial carcinoma (UCEC). While, significantly lower expression was observed in kidney chromophobe $(\mathrm{KICH})$ and thyroid carcinoma (THCA).

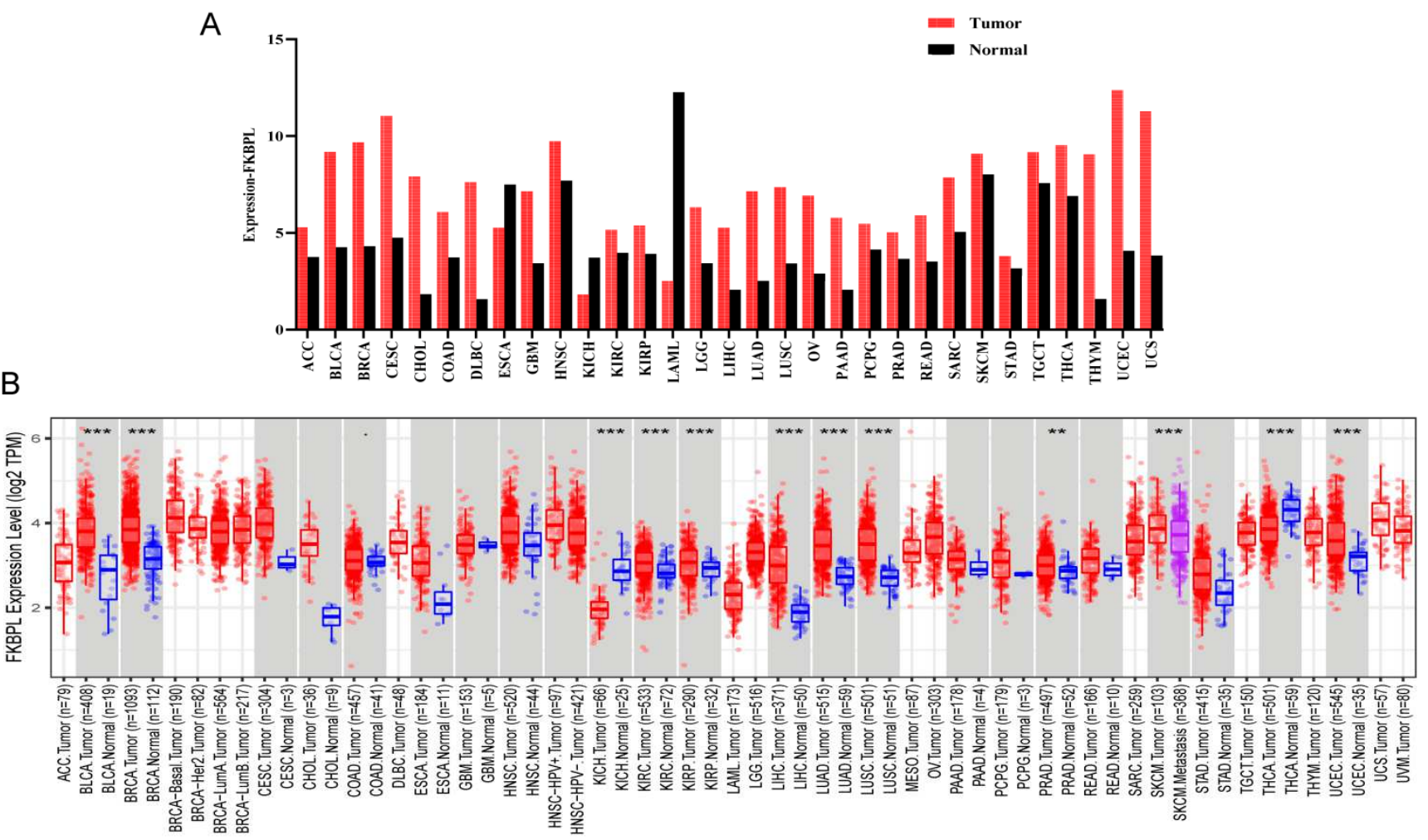

B

Figure 1. The expression levels of FKBPL in different cancer types. (A) Human FKBPL expression levels in different tumor types were determined by GEPIA2 database. (B) Human FKBPL expression levels in different tumor types were determined by TIMER2. ${ }^{* * *} p<.001,{ }^{* *} p<.01,{ }^{*} p<.05$.

\subsection{Overexpression of FKBPL in Hepatocellular Carcinoma}

We downloaded liver cancer datasets in GEO to explore the expression of FKBPL in HCC (2A-K), and details of these datasets are shown in Table 1. The results show that FKBPL was significantly overexpressed in HCC compared with matched nontumor tissues in GSE25097(A), GSE55092(B), GSE84598(C), GSE87630(D), GSE45436(E), GSE45267(F),
GSE76297(G), GSE76311(H), GSE60502(I), GSE121248(J), GSE46408(K). Then, the significantly high expression of FKBPL in HCC was verified again using TCGA data from GEPIA2 database (Figure 2L). Then, the relationship between the expression level of FKBPL and the stage of HCC was performed by GEPIA1database (Figure 2M), and we found that the expression level of FKBPL was significantly correlated with the HCC clinical stage. Additionally, meta-analysis of FKBPL expression in 4 analyses was 
performed in Oncomine database (Figure 2N). We found that FKBPL was significantly upregulated in hepatocellular

carcinoma compared with that in normal liver tissues.

Table 1. Details of GEO series included in this analysis.

\begin{tabular}{llll}
\hline GSE Series & Tumor & Nontumor & Platform \\
\hline GSE25097 & 268 & 243 & GPL10687 Rosetta/Merck Human RSTA Affymetrix 1.0 microarray, Custom CDF \\
GSE55092 & 39 & 81 & GPL570 [HG-U133_Plus_2] Affymetrix Human Genome U133 Plus 2.0 Array \\
GSE84598 & 22 & 22 & GPL10558 Illumina HumanHT-12 V4.0 expression beadchip \\
GSE87630 & 64 & 30 & GPL6947 Illumina HumanHT-12 V3.0 expression beadchip \\
GSE45436 & 97 & 37 & GPL570[HG-U133_Plus_2] Affymetrix Human Genome U133 Plus 2.0 Array \\
GSE45267 & 46 & 41 & GPL570[HG-U133_Plus_2] Affymetrix Human Genome U133 Plus 2.0 Array \\
GSE76297 & 61 & 58 & GPL17586 [HTA-2_0] Affymetrix Human Transcriptome Array 2.0 [transcript (gene) version] \\
GSE76311 & 62 & 59 & GPL17586 [HTA-2_0] Affymetrix Human Transcriptome Array 2.0 [transcript (gene) version] \\
GSE60502 & 18 & 18 & GPL96 [HG-U133A] Affymetrix Human Genome U133A Array \\
GSE121248 & 70 & 37 & GPL570 [HG-U133_Plus_2] Affymetrix Human Genome U133 Plus 2.0 Array \\
GSE46408 & 6 & 6 & GPL4133 Agilent-014850 Whole Human Genome Microarray 4x44K G4112F (Feature Number version) \\
\hline
\end{tabular}
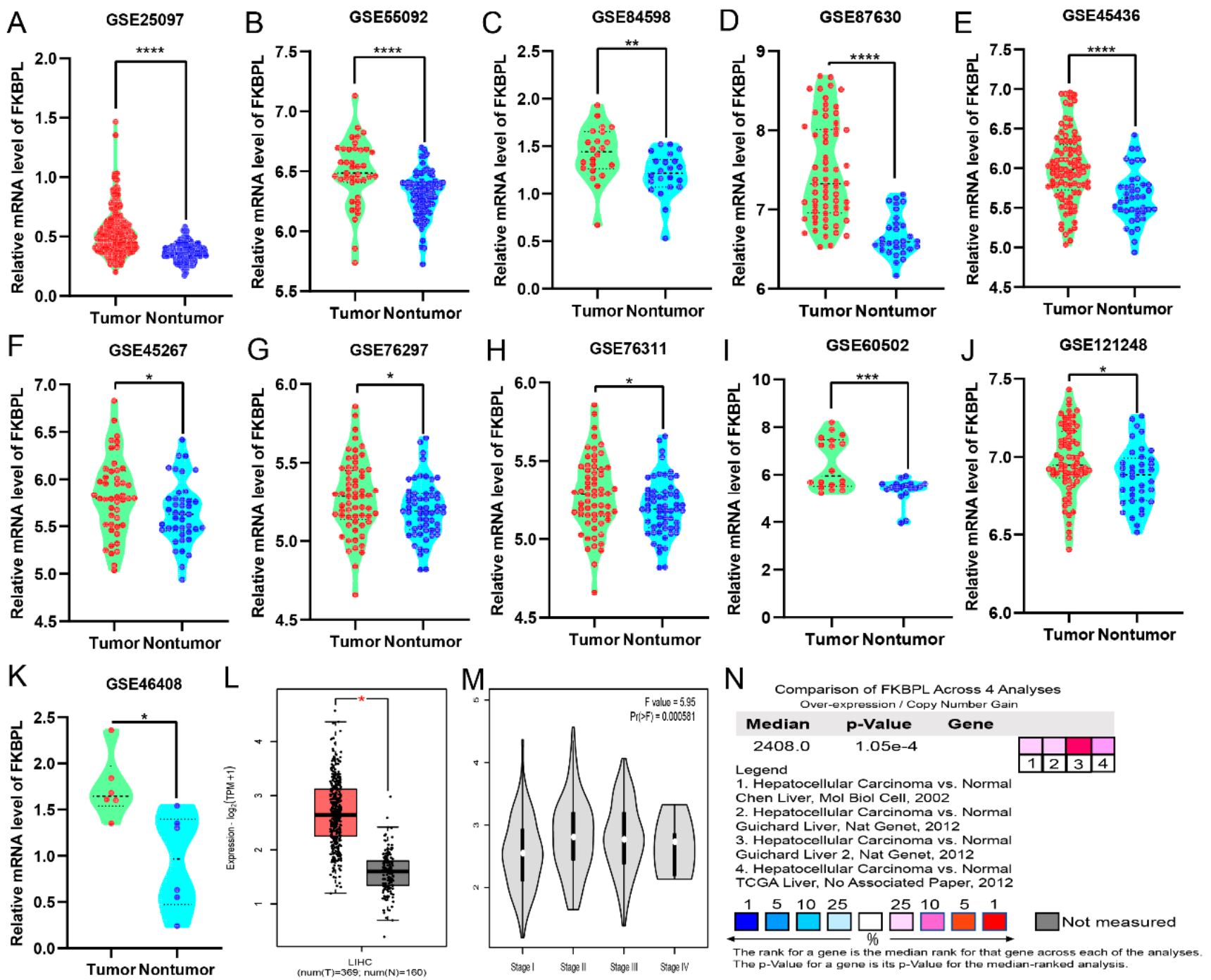

Figure 2. FKBPL mRNA expression levels in hepatocellular carcinoma (HCC). (A-L) FKBPL mRNA expression levels between tumor and nontumor tissues in HCC patients in GEO database series including GSE25097(A), GSE55092(B), GSE84598(C), GSE87630(D), GSE45436(E), GSE45267(F), GSE76297(G), GSE76311(H), GSE60502(I), GSE121248(J), GSE46408(K) and GEPIA2 database (L). (M) Meta-analysis of FKBPL mRNA expression levels in 7 analyses using Oncomine database. ${ }^{* * *} p<0.001,{ }^{* *} p<0.01,{ }^{*} p<0.05$. 


\subsection{Relationship Between High Expression of FKBPL and Prognosis of HCC Patients}

To better understand the relevance and underlying mechanisms of FKBPL expression in HCC, we investigated the relationship between the FKBPL expression and clinical characteristics of HCC patients in GEPIA2 databases (Figure 3A-B) and TIMER database (Figure 3C-G). The results show that overexpression of FKBPL was associated with worse OS and PFS in HCC patients $(\mathrm{P}<0.05)$. Specifically, high FKBPL mRNA expression was correlated with worse prognosis in male and female HCC patients as well as three types of races in the world and purity of tumor $(\mathrm{P}<0.05)$. In addition, overexpression of FKBPL was correlated with worse prognosis stages of HCC patients and age of HCC, but it was not statistically significant. All of above results suggested that FKBPL expression level can impact the prognosis in $\mathrm{HCC}$ patients.
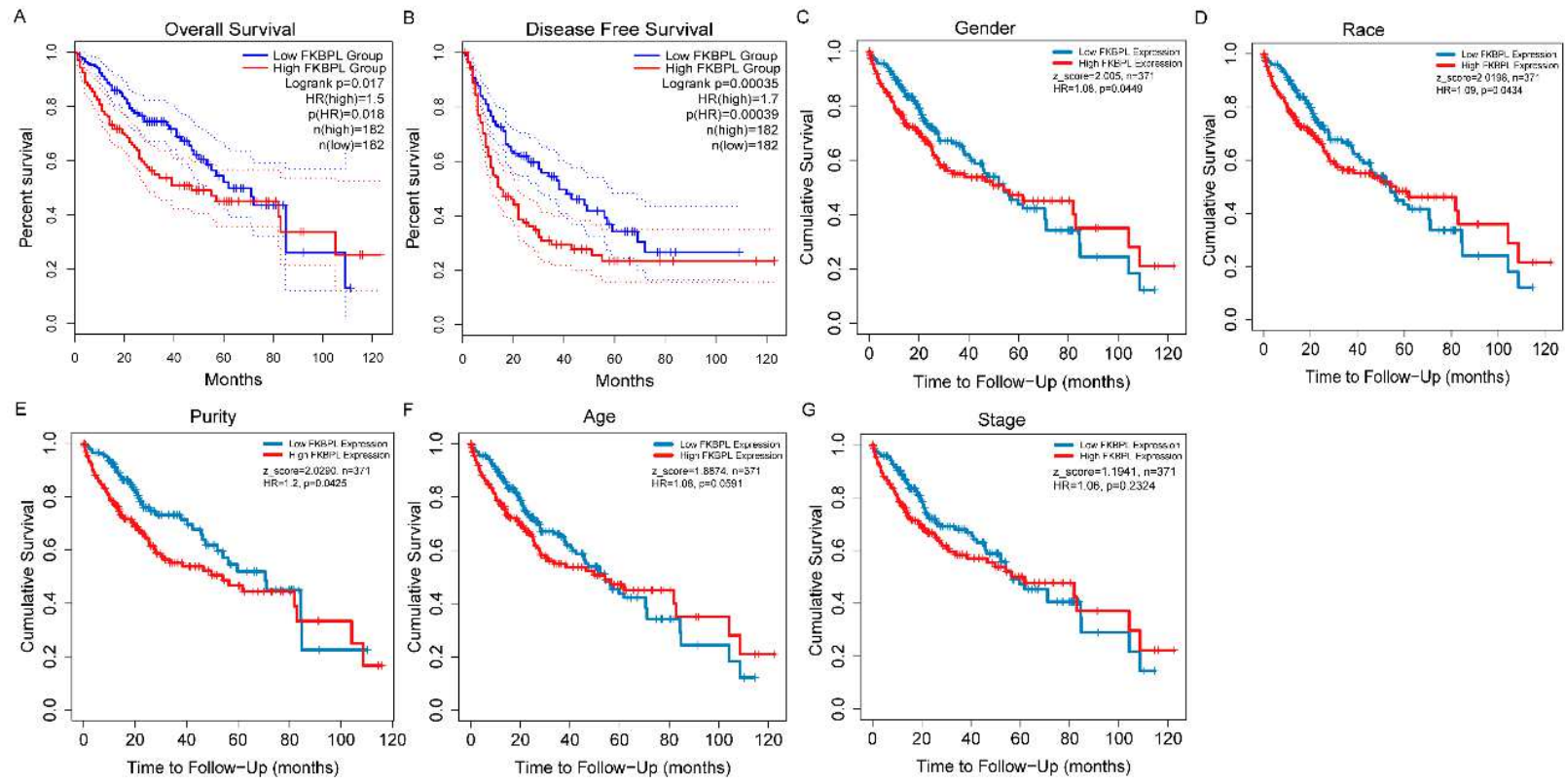

Figure 3. Survival analysis of FKBPL expression and clinical characteristics of HCC patients. Overall survival (A) and disease-free survival (B) of HCC patients in GEPIA2 database. Subgroup analyses of overall survival comparison in different population [gender $(C)$, race $(D)$, purity $(E)$, age $(F)$ and stage $(G)]$ with FKBPL expressed in HCC patients through TIMER2.0 database.

\subsection{Correlation FKBPL Expression with Immune Infiltration Level in $\mathrm{HCC}$}

As tumor-infiltrating lymphocytes can serve as a predictor of survival in cancers [29], we investigated the correlation FKBPL expression with immune infiltration levels in HCC, and then we analyzed correlation FKBPL expression with immune infiltration cells (Figure 4). After purity adjusted, FKBPL expression had significant correlations with infiltrating levels of CD4+ T cells, B cells, dendritic cells, neutrophils, macrophages, T cell NK cells, Monocyte, CD8+ $\mathrm{T}$ cells, and regulatory T cell (Treg) in HCC(A-I). In addition, since cancer associated fibroblast, hematopoietic stem cell, common lymphoid progenitor, myeloid derived suppressor cells (MDSC), granulocyte-monocyte progenitor, $\mathrm{T}$ cell follicular helper, endothelial cell, and mast cell can affect tumor progression, invasion, metastasis, and prognosis by affecting tumor microenvironment[30-32], we analyzed the relationship between the FKBPL expression and tumor microenvironment (Figure 4J-Q). The results show that FKBPL expression had a strong negative correlation with cancer associated fibroblast (Figure 4J), hematopoietic stem cell (Figure 4K), granulocyte-monocyte progenitor (Figure
4N), and endothelial cell (Figure 4P). While, FKBPL expression was significantly positively correlated with common lymphoid progenitor (Figure 4L), MDSC (Figure 4M), T cell follicular helper (Figure 4O), and mast cell (Figure $4 Q$ ). These findings strongly suggest that FKBPL plays a specific role in immune infiltration in HCC.

\subsection{Correlation Analysis Between FKBPL Expression and Immune Markers}

Through TIMER and GEPIA2 databases, we analyzed the correlations between FKBPL expression and immune marker genes of different immune cells, included B cells, CD8+ T cells, $\mathrm{T}$ cells (general), TAMs, dendritic cells, monocytes, natural killer cells (NK cells), M1 and M2 macrophages, and neutrophils in HCC, as well as the different functional T cells, such as regulatory $\mathrm{T}$ cells (Treg), Follicular helper T cells (Tfh cells), Th1 cells, Th2 cells, and Th17 cells, and exhausted T cells (Figure 5, Table 2). After the correlation adjustment by purity, the results revealed the FKBPL expression level was significantly positive correlated with most immune marker sets of various immune cells and different $\mathrm{T}$ cells in HCC. Interestingly, we found that the expression levels of most markers of CD8+ T cell, TAMs, Treg, Th1 cells, Th2 cells, and 
exhausted $\mathrm{T}$ cells have strong correlations with FKBPL expression in $\mathrm{HCC}((\mathrm{P}<0.0001$; Figure 5, Table 2$)$. Specifically, we showed CD8B of CD8+ T cell, CD68 of TAMs, TGF $\beta$ of
Tregs, IFN- $\gamma$ of Th1 cells, STAT5A of Th2 cells, PD-1, CTLA4, and LAG3 of exhausted $\mathrm{T}$ cells are significantly positive correlate with LAYN expression in HCC $(\mathrm{P}<0.0001)$.
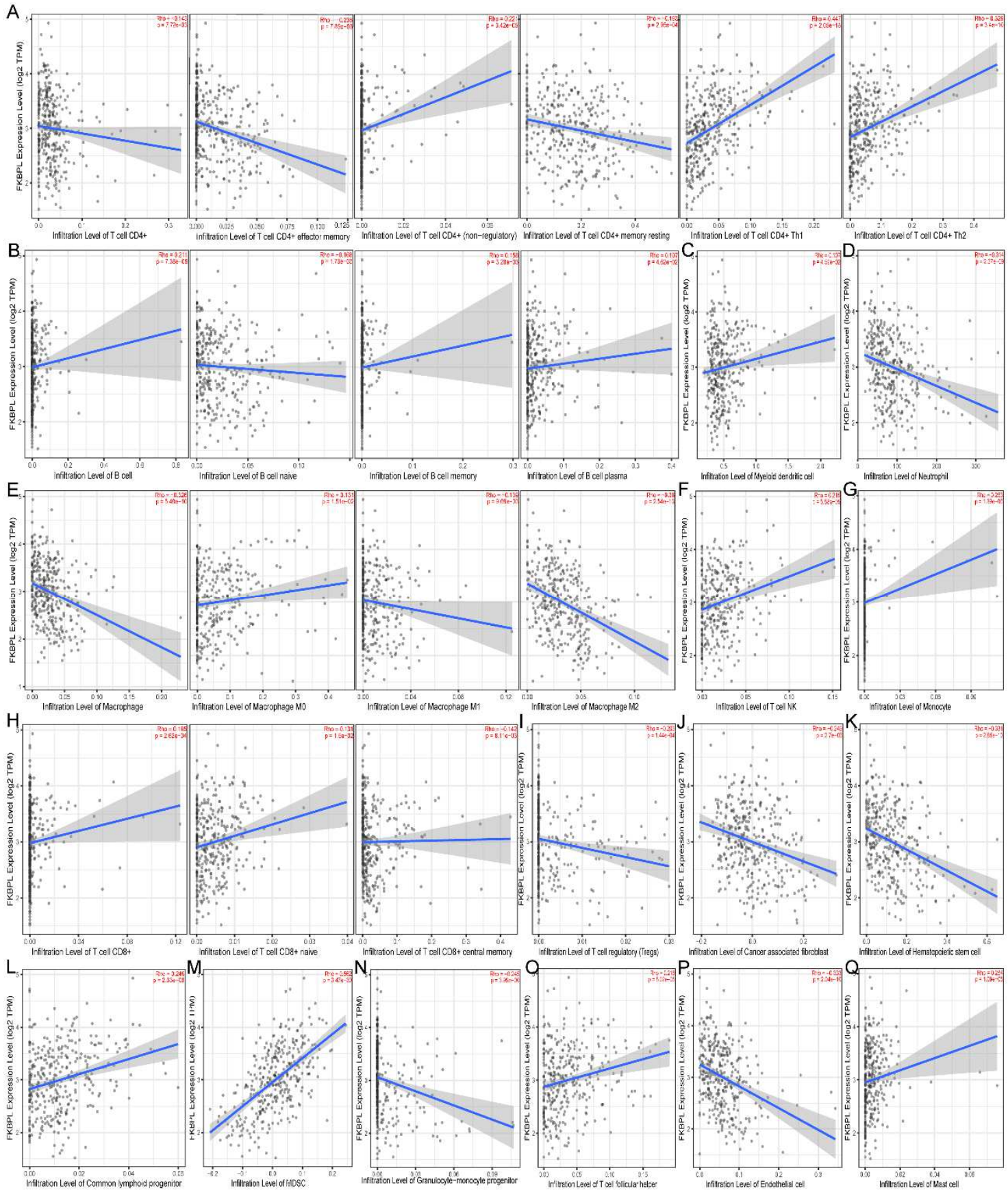

HCC, hepatocellular carcinoma; MDSC, myeloid derived suppressor cells; Th, T helper cell; Tfh, Follicular helper T cell; Treg, regulatory T cell.

Figure 4. Correlation of FKBPL expression with immune infiltration level in HCC. (A)FKBPL expression is significantly negative correlations with infiltrating levels of $C D 4+T$ cell, effector memory $C D 4+T$ cell and memory resting $C D 4+T$ cell, and has significant positive correlations with infiltrating levels of Th1 $C D 4+T$ cell, non-regulatory $C D 4+T$ cell and Th2 CD4 $T$ T cell in HCC. (B) FKBPL expression is significantly negatively related to the infiltrating levels of naive $B$ cell and has significant positive correlations with infiltrating levels of $B$ cell, memory $B$ cell and plasma $B$ cell in $H C C$. (C) FKBPL expression is significantly positively related to myeloid dendritic cell. (D) FKBPL expression is significantly negatively related to neutrophil. (E) FKBPL expression is significantly positively related to macrophage $\mathrm{MO}$ and has significant negative correlations with infiltrating levels of macrophage, macrophage M1 and macrophage M2. (F) FKBPL expression is significantly positively related to NK T cell. (G) FKBPL expression is significantly positively related to monocyte. (H) FKBPL expression has significant positive correlations with infiltrating levels of CD8+T cell, naive CD8 $+T$ cell and central memory CD8+T cell. (I) FKBPL expression is significantly negatively related to Tregs. (J-Q) FKBPL expression is significantly negatively related to cancer associated fibroblast(J), negatively related to hematopoietic stem cell(K), positively related to common lymphoid progenitor $(L)$, positively related to MDSC (M), negatively related to granulocyte-monocyte progenitor $(N)$, positively related to $T$ cell follicular helper $(O)$, negatively related to endothelial cell $(P)$ and positively related to mast $\operatorname{cell}(Q)$. 
It is reported that dendritic cells can promote tumor growth and metastasis by Regulating Treg cells and CD8+T cells [33]. In order to further explore the mechanism of FKBPL expression in HCC cells migration, we focused on correlation between FKBPL expression and dendritic cells, Treg cells and $\mathrm{CD} 8+\mathrm{T}$ cells. We found high FKBPL expression relates to high infiltration level of dendritic cells in liver, and dendritic cells markers such as HLA-DPB1, HLA-DPB1, HLA-DRA, and BDCA-4(NRP1) show significant correlations with FKBPL expression. These results further reveal that there is a strong relationship between FKBPL and dendritic cells infiltration. In addition, for Treg cells, FKBPL is a negative correlation with TGFB1 in HCC (Figure 5J), as well as a significant positive correlation between FKBPL and CD8+ T cell of CD8A and CD8B (Figure 5B). The above results show that FKBPL may be a crucial factor to promote tumor growth and metastasis by mediating dendritic cells.

In addition, we found that there are significant correlations between FKBPL and marker genes of $\mathrm{T}$ cell exhaustion (Figure 5O). As the crucial gene that regulates $\mathrm{T}$ cell exhaustion, PD-1, CTLA4, TIM-3, and GZMB, have strong positive correlation with FKBPL expression, suggesting that high FKBPL expression plays important roles in PD-1, CTLA4, TIM-3, and GZMB mediating $\mathrm{T}$ cell exhaustion. Those results further suggest that FKBPL plays a vital role in immune escape in the HCC microenvironment.

Table 2. Correlation analysis between FKBPL and relate genes and markers of immune cells in HCC through TIMER2.0.

\begin{tabular}{|c|c|c|c|c|c|}
\hline \multirow[t]{2}{*}{ Description } & \multirow[t]{2}{*}{ Gene markers } & \multicolumn{2}{|l|}{ None } & \multicolumn{2}{|l|}{ Purity } \\
\hline & & cor & $\mathbf{p}$ & cor & $\mathbf{p}$ \\
\hline \multirow[t]{2}{*}{ B cell } & CD19 & 0.065 & $2.08 \mathrm{E}-01$ & 0.147 & $6.33 \mathrm{E}-03$ \\
\hline & CD79A & -0.025 & $6.30 \mathrm{E}-01$ & 0.097 & 7.19E-02 \\
\hline \multirow[t]{2}{*}{ CD8+ T cell } & CD8A & 0.004 & $9.34 \mathrm{E}-01$ & 0.127 & $1.84 \mathrm{E}-02$ \\
\hline & CD8B & 0.094 & $7.10 \mathrm{E}-02$ & 0.211 & 8.07E-05 \\
\hline \multirow[t]{2}{*}{ T cell (general) } & CD3D & 0.054 & $3.01 \mathrm{E}-01$ & 0.176 & $1.04 \mathrm{E}-03$ \\
\hline & CD3E & -0.047 & $3.71 \mathrm{E}-01$ & 0.093 & $8.33 \mathrm{E}-02$ \\
\hline \multirow[t]{3}{*}{ TAM } & CCL2 & -0.027 & $6.00 \mathrm{E}-01$ & 0.073 & $1.77 \mathrm{E}-01$ \\
\hline & CD68 & 0.067 & $1.96 \mathrm{E}-01$ & 0.178 & $9.09 \mathrm{E}-04$ \\
\hline & IL10 & -0.027 & $6.00 \mathrm{E}-01$ & 0.071 & $1.86 \mathrm{E}-01$ \\
\hline \multirow[t]{6}{*}{ Dendritic cell } & HLA-DPB1 & 0.041 & 4.27E-01 & 0.149 & $5.44 \mathrm{E}-03$ \\
\hline & HLA-DQB1 & 0.04 & $4.43 \mathrm{E}-01$ & 0.147 & $6.31 \mathrm{E}-03$ \\
\hline & HLA-DRA & 0.007 & 8.91E-01 & 0.109 & $4.31 \mathrm{E}-02$ \\
\hline & HLA-DPA1 & -0.024 & $6.40 \mathrm{E}-01$ & 0.066 & $2.23 \mathrm{E}-01$ \\
\hline & BDCA-1(CD1C) & -0.064 & $2.21 \mathrm{E}-01$ & 0.02 & $7.41 \mathrm{E}-01$ \\
\hline & CD11c (ITGAX) & -0.001 & $9.79 \mathrm{E}-01$ & 0.098 & $6.90 \mathrm{E}-02$ \\
\hline \multirow[t]{2}{*}{ Monocyte } & CD86 & 0.036 & $4.95 \mathrm{E}-01$ & 0.16 & $2.80 \mathrm{E}-03$ \\
\hline & CD115 (CSF1R) & 0.001 & $9.77 \mathrm{E}-01$ & 0.122 & $2.31 \mathrm{E}-02$ \\
\hline \multirow[t]{7}{*}{ Natural killer cell } & KIR2DL1 & -0.027 & $6.03 \mathrm{E}-01$ & -0.059 & 2.77E-01 \\
\hline & KIR2DL3 & 0.085 & $1.00 \mathrm{E}-01$ & 0.108 & $4.42 \mathrm{E}-02$ \\
\hline & KIR2DL4 & 0.107 & $3.91 \mathrm{E}-02$ & 0.14 & $9.12 \mathrm{E}-03$ \\
\hline & KIR3DL1 & -0.03 & $5.71 \mathrm{E}-01$ & -0.016 & 7.69E-01 \\
\hline & KIR3DL2 & 0.061 & $2.38 \mathrm{E}-01$ & 0.133 & $1.34 \mathrm{E}-02$ \\
\hline & KIR3DL3 & 0.018 & $7.27 \mathrm{E}-01$ & 0.027 & $6.18 \mathrm{E}-01$ \\
\hline & KIR2DS4 & 0.017 & 7.47E-01 & 0.002 & $9.69 \mathrm{E}-01$ \\
\hline \multirow[t]{3}{*}{ M1 Macrophage } & INOS (NOS2) & -0.129 & $1.32 \mathrm{E}-02$ & -0.137 & $1.10 \mathrm{E}-02$ \\
\hline & IRF5 & 0.149 & $4.12 \mathrm{E}-03$ & 0.141 & $8.56 \mathrm{E}-03$ \\
\hline & COX2(PTGS2) & -0.133 & $1.01 \mathrm{E}-02$ & -0.04 & $4.57 \mathrm{E}-01$ \\
\hline \multirow[t]{2}{*}{ M2 Macrophage } & CD163 & -0.098 & $5.86 \mathrm{E}-02$ & -0.007 & $8.96 \mathrm{E}-01$ \\
\hline & VSIG4 & -0.084 & $1.05 \mathrm{E}-01$ & 0.003 & $9.61 \mathrm{E}-01$ \\
\hline \multirow{2}{*}{ Neutrophils } & CD11b (ITGAM) & 0.082 & $1.15 \mathrm{E}-01$ & 0.146 & $6.55 \mathrm{E}-03$ \\
\hline & CCR7 & -0.12 & $2.06 \mathrm{E}-02$ & -0.007 & $9.00 \mathrm{E}-01$ \\
\hline \multirow[t]{2}{*}{ Tfh } & BCL6 & 0.114 & $2.83 \mathrm{E}-02$ & 0.136 & $1.16 \mathrm{E}-02$ \\
\hline & IL21 & -0.003 & $9.51 \mathrm{E}-01$ & 0.049 & $3.60 \mathrm{E}-01$ \\
\hline \multirow[t]{4}{*}{ Treg } & FOXP3 & -0.051 & $3.24 \mathrm{E}-01$ & -0.015 & 7.77E-01 \\
\hline & CCR8 & -0.039 & $4.53 \mathrm{E}-01$ & 0.028 & $6.07 \mathrm{E}-01$ \\
\hline & STAT5B & 0.122 & $1.86 \mathrm{E}-02$ & 0.078 & $1.48 \mathrm{E}-01$ \\
\hline & TGF $\beta$ (TGFB1) & 0.082 & $1.13 \mathrm{E}-01$ & -0.41 & $1.98 \mathrm{E}-15$ \\
\hline \multirow[t]{5}{*}{ Th1 } & T-bet (TBX21) & -0.043 & $4.07 \mathrm{E}-01$ & 0.038 & 4.82E-01 \\
\hline & STAT4 & -0.032 & $5.33 \mathrm{E}-01$ & 0.033 & $5.47 \mathrm{E}-01$ \\
\hline & STAT1 & 0.056 & $2.85 \mathrm{E}-01$ & 0.116 & $3.16 \mathrm{E}-02$ \\
\hline & IFN- $\gamma($ IFNG) & 0.149 & 4.03E-03 & 0.224 & $2.70 \mathrm{E}-05$ \\
\hline & TNF- $\alpha(\mathrm{TNF})$ & 0.024 & $6.46 \mathrm{E}-01$ & 0.141 & 8.93E-03 \\
\hline Th2 & GATA3 & -0.029 & $5.72 \mathrm{E}-01$ & 0.08 & $1.38 \mathrm{E}-01$ \\
\hline
\end{tabular}




\begin{tabular}{llllll}
\hline Description & Gene markers & None & & Purity \\
\hline & & cor & p & cor & p \\
\hline & STAT6 & -0.023 & $6.52 \mathrm{E}-01$ & -0.054 & $3.16 \mathrm{E}-01$ \\
& STAT5A & 0.189 & $2.59 \mathrm{E}-04$ & 0.25 & $2.54 \mathrm{E}-06$ \\
Th17 & IL13 & 0.082 & $1.16 \mathrm{E}-01$ & 0.063 & $2.44 \mathrm{E}-01$ \\
& STAT3 & -0.077 & $1.37 \mathrm{E}-01$ & -0.049 & $3.69 \mathrm{E}-01$ \\
T cell exhaustion & IL17A & -0.003 & $9.53 \mathrm{E}-01$ & 0 & $9.96 \mathrm{E}-01$ \\
& PD-1 (PDCD1) & 0.09 & $8.33 \mathrm{E}-02$ & 0.223 & $2.91 \mathrm{E}-05$ \\
& CTLA4 & 0.078 & $1.33 \mathrm{E}-01$ & 0.187 & $4.68 \mathrm{E}-04$ \\
& LAG3 & 0.114 & $2.77 \mathrm{E}-02$ & 0.182 & $6.85 \mathrm{E}-04$ \\
& TIM-3 (HAVCR2) & 0.008 & $8.72 \mathrm{E}-01$ & 0.126 & $1.92 \mathrm{E}-02$ \\
& GZMB & 0.092 & $7.53 \mathrm{E}-02$ & 0.153 & $4.47 \mathrm{E}-03$ \\
& PDL1(CD274) & 0.013 & $8.09 \mathrm{E}-01$ & 0.046 & $3.96 \mathrm{E}-01$ \\
\hline
\end{tabular}

HCC, hepatocellular carcinoma; TAM, tumor-associated macrophage; Th, T helper cell; Tfh, Follicular helper T cell; Treg, regulatory T cell; Cor, R value of Spearman's correlation; None, correlation without adjustment. Purity, correlation adjusted by purity.
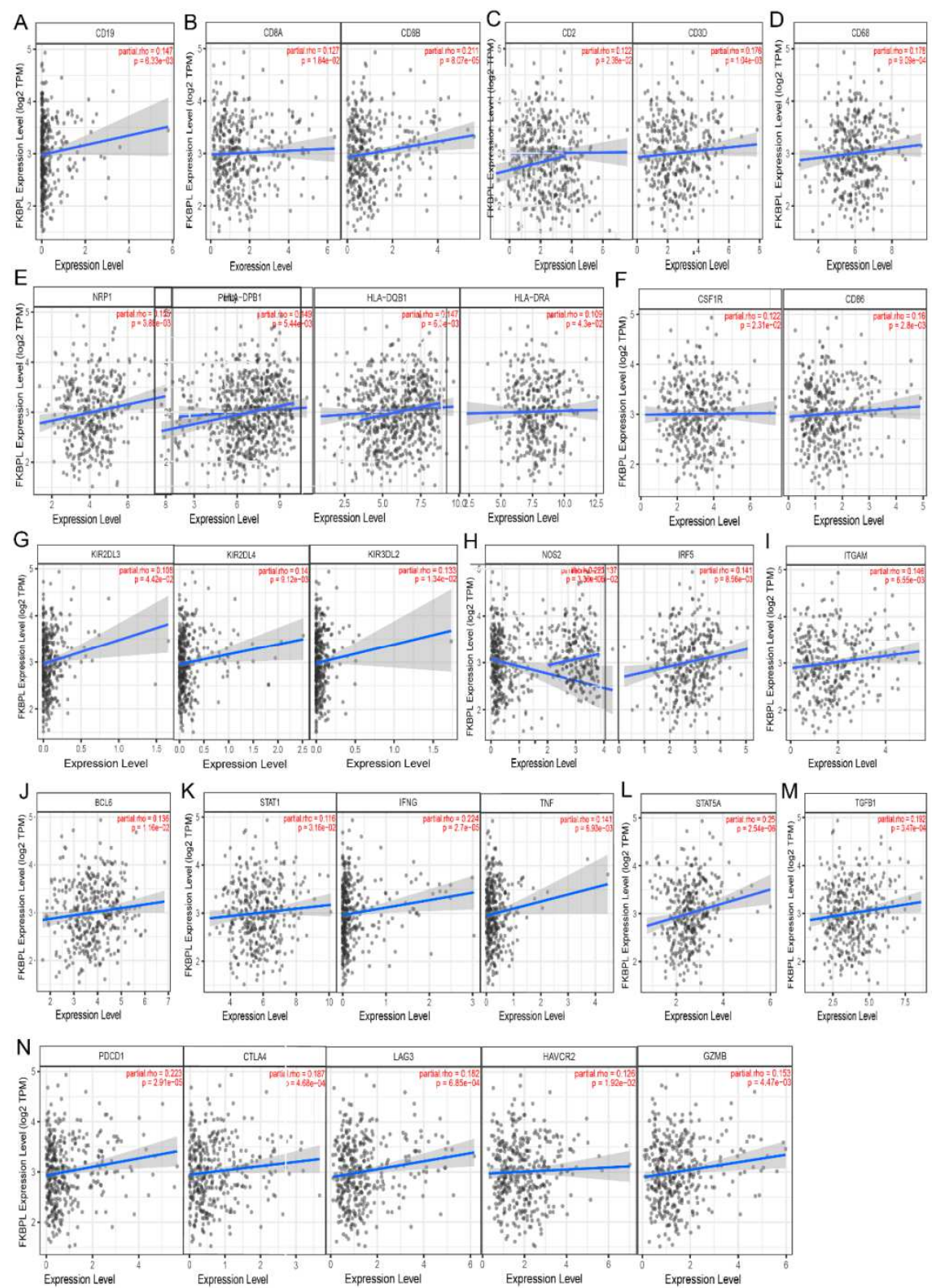

Figure 5. FKBPL expression correlated with immune markers in HCC. Markers include: $C D 19$ of B cell(A); $C D 8 A$ and $C D 8 B$ of CD8+T cell(B); $C D 2$ and CD3D of T cell (general)(C); CD68 of TAM(D); BDCA-4(NRP1), HLA-DPB1, HLA-DQB1 and HLA-DRA of dendritic cell(E); CD115 (CSF1R) and CD86 of monocyte(F); KIR2DL3, KIR2DL4 and KIR3DL2 of natural killer cell(G); INOS (NOS2) and IRF5 of M1 macrophage(H); CD11b (ITGAM) of neutrophils(I);

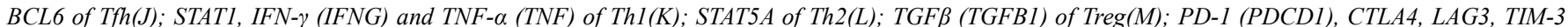
(HAVCR2) and GZMB of(N). 


\subsection{PPI and GO Biological Process Enrichment}

PPI (protein-protein interaction) analysis using STRING database revealed that 10 genes including PRRT1, ANKRD49, RBCK1, CSNK1D, HSP90AA1, HSP90AB1, GTSE1, CDKN1A, AVPR2, and EGFL8 were interacted with FKBPL (Figure 6A). Then, the top 500 genes that expressed similar FKBPL in HCC were downloaded from the GEPIA2 database (Supplementary material table S1). Enrichment analysis with these genes through Metascape showed that the genes similar to FKBPL in HCC were mainly enriched in apoptotic signaling pathway, regulation of cellular response to stress, regulation of mRNA metabolic process, regulation of myeloid cell differentiation, cell death in response to oxidative stress, mRNA surveillance pathway, epithelial cell apoptotic process, PIP3 activates AKT signaling, et al. (Figure 6B-C).
A

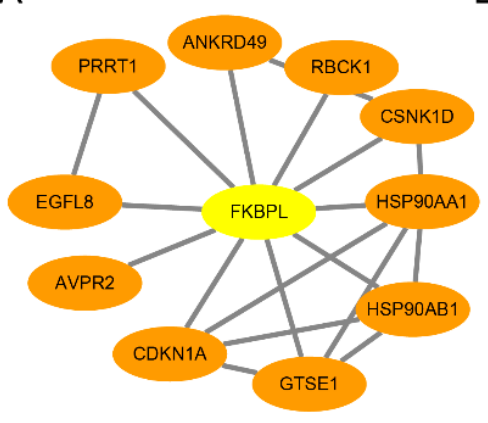

C

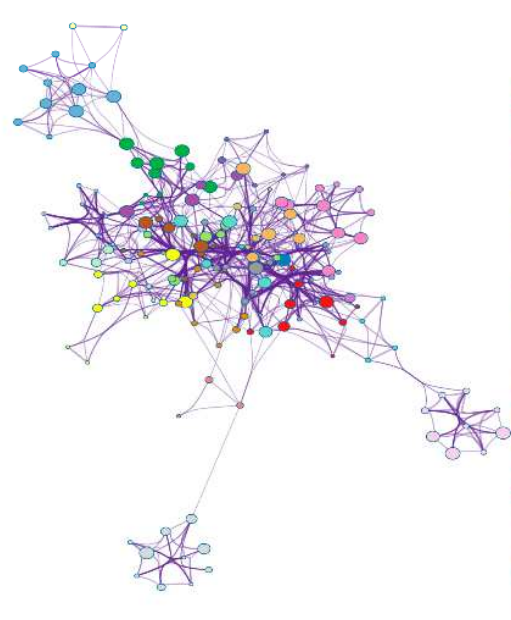

B

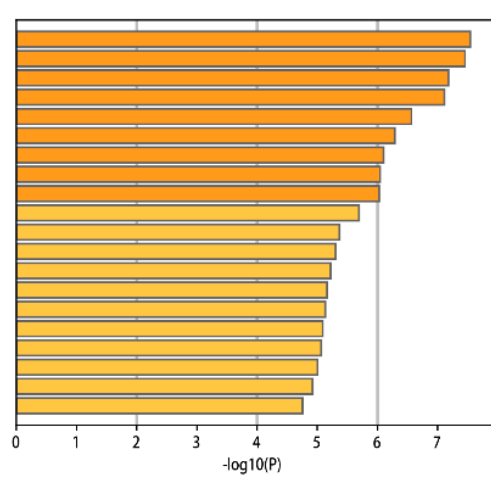

GO:0097190: apoptotic signaling pathway 60:0080135: regulation of cellular response to stres 0:1903311: regulation of mRNA metabolic process M250: PID HIF 1 TFPATHWAY 0:0043620: regulation of DNA-templated transcription in response to stress 60:0045637: regulation of myeloid cell differentiation 0:0031400: negative regulation of protein modification process 0:0036473: cell death in response to oxidative stress G0:0031331: positive regulation of cellular catabolic process R. KISA-2559585: Oncogene Induced Senescence GO:1904019: epithelial cell apoptotic process R-HSA-8953854: Metabolism of RNA GO:0038066: p38MAPK cascade GO:0071496: cellular response to ex GO:00060972: Listone modfificication GO:0097193: intrinsic apoptotic signaling pathway R-HSA-1257604: PIP3 activates AKT signaling

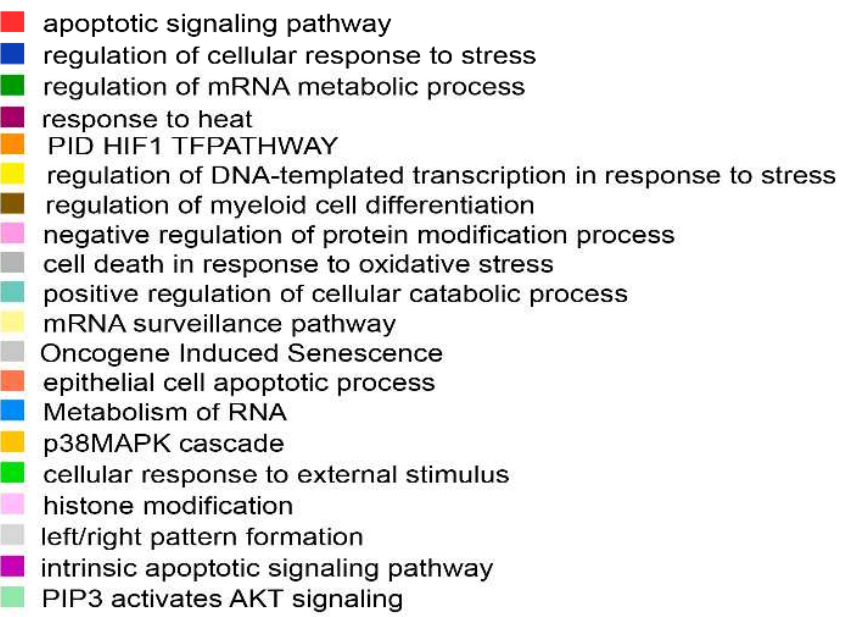

Figure 6. Protein-protein interaction of FKBPL using String analysis (A); The visualized networks of functional enrichment of the top 500 genes that expressed similar FKBPL in HCC, then each node represents an enriched term and is colored first by its p-value(B) and by its cluster ID (C).

\section{Discussion}

FKBPL (as known as DIR1, NG7, WISp39), a member of FKBP family (FKBPs), plays an important role on development of various aspects of lymphocytes especially $\mathrm{T}$ cells and can bind to immunosuppressant drugs [34], which is a immunophilin family and its members have fundamental roles in the regulation of signalling pathways involved in inflammation, adaptive immune responses, cancer and developmental biology [35]. Up to now, immunophilin-based immunosuppressant drugs have been pivotal in the lives of organ transplant recipients by improving graft rejection response rates. Through blocking the activity of programmed death-ligand 1 (PD-L1) on tumour cells or its receptor, programmed cell death protein 1 (PD-1) on $\mathrm{T}$ cells, immune checkpoint inhibitors have emerged as a front line therapy for cancer, including HCC [36]. In addition, previous studies have demonstrated that, FKBPL is also secreted from numerous cell types, including endothelial cells and has taken a different approach to develop drug therapies, the targeted therapy based on FKBPL will have a broad prospect in cancer treatment [37]. However, the relationships among FKBPL expression, prognosis, immune infiltration, and $\mathrm{T}$ cells function in HCC have not been comprehensively investigated.

In this study, we obtained and analyzed extensive tumor samples using several large databases comprised a large number of tumor and normal samples, and after repeated verification, it was found that FKBPL was significantly upregulated in HCC tumors compared with normal livers. Additionally, the expression level of FKBPL was correlated with prognosis in HCC. Further studies showed that FKBPL expression was significantly correlated with the level of immune infiltration in HCC. We then confirmed that FKBPL interacts significantly with most functional $\mathrm{T}$ cells in $\mathrm{HCC}$, including exhaustion $\mathrm{T}$ cell. Therefore, we assumed that 
FKBPL is emerging as a prognostic biomarker in HCC, and provide a new direction for understanding the correlation between FKBPL, immune infiltration, and T cell function. As another member of the FKBP family, FKBP52 has been shown to be significantly highly expressed in HCC, and it serves as a potential biomarker associated with different $\mathrm{HCC}$ clinical stages (with a significant reduction in third-stage HCC expression) [38]. And previous studies that FKBP11 has been demonstrated to be highly expressed and serves as biomarker in HCC [39]. FKBP12 was found to be overexpressed in malignant vascular endothelium [40] and childhood astrocytomas [41]. Some members of FKBPs were overexpressed in cancers, such as FKBP38 in neuroblastoma cells [42], FKBP52 in estrogen receptor-positive breast cancer tissues [43] and prostate cancer [44], FKBP51 in glioma [45] and prostate cancer [46], FKBP51 in lymphoma [47] and melanoma [48], and FKBP65 in colorectal cancer [49]. As another member of the FKBP family, the expression of FKBPL and its relationship with prognosis in HCC are unknown. Our study found that FKBPL is significantly highly expressed in $\mathrm{HCC}$, and it can serve as a potential prognostic biomarker in HCC. Due to the excellent potential of FKBPL for HCC prognosis and the subsequent examination of the correlation between FKBPL expression and the clinical characteristics of $\mathrm{HCC}$ patients, we found that the survival rate of HCC patients with high FKBPL expression was significantly correlated with race, gender and tumor purity of HCC patients, as well as age tumor stage, but $\mathrm{p}$ values were not statistically significant (Figure 3). In addition, we found that the expression level of FKBPL was significantly correlated with the tumor stage of HCC (Figure 2M). Over the past few years, some patients with metastatic cancer have benefited from treatment with immune checkpoint inhibitors and considering the critical role of FKBPs in the immune system and its outstanding prognostic value for cancer, we analyzed the relationship between FKBPL and the level of immune infiltration in HCC (Figure 4-5). The results showed that high expression level of FKBPL is significantly correlated with high immune infiltration levels of most of the immune cell populations including CD4 $+\mathrm{T}$ cells, B cells, dendritic cells, neutrophils, macrophages, T cell NK cells, Monocyte, CD8+ T cells, and regulatory $\mathrm{T}$ cell (Treg) in HCC. Further studies on the correlation between FKBPL and immune cell genetic markers have shown that FKBPL interacts with not only most immune cells, but also various functional T cells, as well as exhausted T cells, Th1 cells, Th2 cells and Tfh cells. Because exhausted $T$ cells play a critical role in immunotherapy, we noted that high expression of FKBPL is positively correlated with multiple key genes in exhausted $T$ cells (PD-1, CTLA4, LAG3, TIM-3, and GZMB) that are currently therapeutic targets or play key roles in immunotherapy. Myeloid-derived suppressor cells (MDSCs) represent the main immunosuppressive population and only exist in pathological conditions including cancer. The tumor microenvironment can promote immature myeloid cells from the bone marrow (BM) tumor site, further inhibit the proliferation and function of CD8 $+\mathrm{T}$ cells, and induce other immunosuppressive cells, such as tumor-related macrophages and regulatory $\mathrm{T}$ cells, which play an important role in influencing the therapeutic effect and prognosis of HCC patients [8]. And tumor-associated macrophages (TAMs) and neoplastic infiltrates neutrophils (TINs) [4], hematopoietic stem cell $[5,6]$, myeloid derived suppressor cells (MDSC) [7, 8], T cell follicular helper [9], endothelial cell[10], and mast cell[11] can affect the prognosis and efficacy of patients chemotherapy and immunotherapy. In our study, an additional finding is the relationship between FKBPL and mast cells, cancer associated fibroblast, hematopoietic stem cell, common lymphoid progenitor, MDSC, granulocyte-monocyte progenitor, T cell follicular helper and endothelial cell in HCC, which may find possible ways to select novel targets and improve immunotherapy efficacy for advanced HCC patients. In addition, FKBPs members have been implicated in protein-protein interactions with the heat shock protein 90 (HSP90), C-terminal calmodulin-binding domains, and a trans-membrane motif. In addition to their well-established roles in T-cell activation with immunosuppressive ligands [14]. Consistent with previous reports, we found that HSP90AA1 and HSP90AB1 had a significant protein-protein interaction with FKBPL through PPI analysis using STRING database. Our findings suggest that FKBPL has dual functions that high expression level of FKBPL is positively correlated with poor prognosis and correlated with immune infiltrates and T cells exhaustion in $\mathrm{HCC}$

The limitations of this study are as follows: Firstly, our results cannot be verified due to the absence of experiments. In order to verify and further understand the role of FKBPL in immune tumors, we plan to compare the effects of checkpoint blockade on FKBPL knockdown model and normal model in vitro and in vivo. Secondly, our study suggests that FKBPL may be associated with immunotherapy responses in $\mathrm{HCC}$, but we currently do not have appropriate population data to support this hypothesis. We plan to collect relevant data of patients receiving immunotherapy in our hospital, hoping to obtain sufficient sample size for survival analysis. Finally, the data used in our study came from public databases, but the quality of the data could not be well evaluated.

\section{Conclusions}

In a nutshell, our findings suggest that FKBPL is a prognosis-related biomarker in HCC. Overexpressed FKBPL correlates with high immune infiltration levels in B cells, CD8+ $\mathrm{T}$ cells, $\mathrm{T}$ cells (general), TAMs, dendritic cells, monocytes, natural killer cells (NK cells), M1 and M2 macrophages, and neutrophils in HCC, as well as the different functional $\mathrm{T}$ cells, such as Tregs, Tfh cells, Th1 cells, Th2 cells, and Th17 cells, and exhausted T cells. Notwithstanding playing crucial role on the development of immunity, FKBPL also highly correlates with exhausted $\mathrm{T}$ cells and is probable a critical regulator promoted T cells exhaustion in HCC. And detecting FKBPL expression may help to predict prognosis and regulating FKBPL expression in exhausted $\mathrm{T}$ cells may provide a new strategy in maximizing immunotherapy 
efficacy for HCC patients.

\section{Abbreviations}

HCC, hepatocellular carcinoma; TAM, tumor-associated macrophage; Th, T helper cell; Tfh, Follicular helper T cell; Treg, regulatory T cell; FKBPL, FKBP Prolyl Isomerase Like; FKBPs, FKBP family

\section{Conflict of Interest}

All the authors do not have any possible conflicts of interest.

\section{Data Availability Statement}

Data downloaded from the GEO database is available from the corresponding author, and the other data supporting the results of this study is available in an open database including GEPIA2 database(http://gepia2.cancer-pku.cn/\#index), TIMER2.0 database (http://timer.cistrome.org/), Oncomine database (https://www.oncomine.org/resource/login.html), and STRING database (https://string-db.org/).

\section{References}

[1] Forner, A., Reig, M. \& Bruix, J. Hepatocellular carcinoma. The Lancet 391, 1301-1314, doi: 10.1016/s0140-6736(18)30010-2 (2018).

[2] De Toni, E. N. et al. Age independent survival benefit for patients with hepatocellular carcinoma (HCC) without metastases at diagnosis: a population-based study. Gut 69, 168-176, doi: 10.1136/gutjnl-2018-318193 (2020).

[3] Jiang, Y., Han, Q. J. \& Zhang, J. Hepatocellular carcinoma: Mechanisms of progression and immunotherapy. World $J$ Gastroenterol 25, 3151-3167, doi: 10.3748/wjg.v25.i25.3151 (2019).

[4] Zhang, H. et al. Tumor-infiltrating Neutrophils is Prognostic and Predictive for Postoperative Adjuvant Chemotherapy Benefit in Patients With Gastric Cancer. Ann Surg 267, 311-318, doi: 10.1097/SLA.0000000000002058 (2018).

[5] Li, C. et al. Prophylactic In Vivo Hematopoietic Stem Cell Gene Therapy with an Immune Checkpoint Inhibitor Reverses Tumor Growth in Syngeneic Mouse Tumor Models. Cancer Res 80, 549-560, doi: 10.1158/0008-5472.CAN-19-1044 (2020).

[6] Merli, P. et al. Role of interferon-gamma in immune-mediated graft failure after allogeneic hematopoietic stem cell transplantation. Haematologica 104, 2314-2323, doi: 10.3324/haematol.2019.216101 (2019).

[7] Tian, X., Shen, H., Li, Z., Wang, T. \& Wang, S. Tumor-derived exosomes, myeloid-derived suppressor cells, and tumor microenvironment. J Hematol Oncol 12, 84, doi: 10.1186/s13045-019-0772-z (2019).

[8] Zhou, J. et al. Hepatoma-intrinsic CCRK inhibition diminishes myeloid-derived suppressor cell immunosuppression and enhances immune-checkpoint blockade efficacy. Gut 67, 931-944, doi: 10.1136/gutjnl-2017-314032 (2018).
[9] Jia, Y. et al. Impaired function of CD4+ T follicular helper (Tfh) cells associated with hepatocellular carcinoma progression. PLoS One 10, e0117458, doi: 10.1371/journal.pone.0117458 (2015).

[10] Ehling, J. \& Tacke, F. Role of chemokine pathways in hepatobiliary cancer. Cancer Lett 379, 173-183, doi: 10.1016/j.canlet.2015.06.017 (2016).

[11] Rohr-Udilova, N. et al. Deviations of the immune cell landscape between healthy liver and hepatocellular carcinoma. Sci Rep 8, 6220, doi: 10.1038/s41598-018-24437-5 (2018).

[12] Khemlina, G., Ikeda, S. \& Kurzrock, R. The biology of Hepatocellular carcinoma: implications for genomic and immune therapies. Mol Cancer 16, 149, doi: 10.1186/s12943-017-0712-x (2017).

[13] Rao, N., Lee, Y. F. \& Ge, R. Novel endogenous angiogenesis inhibitors and their therapeutic potential. Acta Pharmacol Sin 36, 1177-1190, doi: 10.1038/aps.2015.73 (2015).

[14] McKeen, H. D. et al. The emerging role of FK506-binding proteins as cancer biomarkers: a focus on FKBPL. Biochem Soc Trans 39, 663-668, doi: 10.1042/BST0390663 (2011).

[15] Todd, N. et al. Role of a novel angiogenesis FKBPL-CD44 pathway in preeclampsia risk stratification and mesenchymal stem cell treatment. $J$ Clin Endocrinol Metab, doi: 10.1210/clinem/dgaa403 (2020).

[16] Tang, Z., Kang, B., Li, C., Chen, T. \& Zhang, Z. GEPIA2: an enhanced web server for large-scale expression profiling and interactive analysis. Nucleic Acids Res 47, W556-W560, doi: 10.1093/nar/gkz430 (2019).

[17] Tung, E. K. et al. Clinicopathological and prognostic significance of serum and tissue Dickkopf-1 levels in human hepatocellular carcinoma. Liver Int 31, 1494-1504, doi: 10.1111/j.1478-3231.2011.02597.x (2011).

[18] Melis, M. et al. Viral expression and molecular profiling in liver tissue versus microdissected hepatocytes in hepatitis B virus associated hepatocellular carcinoma. Journal of Translational Medicine 12, doi: 10.1186/s12967-014-0230-1 (2014).

[19] Funk, K. et al. BAX Redistribution Induces Apoptosis Resistance and Selective Stress Sensitivity in Human HCC. Cancers (Basel) 12, doi: 10.3390/cancers12061437 (2020).

[20] Woo, H. G. et al. Integrative analysis of genomic and epigenomic regulation of the transcriptome in liver cancer. Nature Communications 8, doi: 10.1038/s41467-017-00991-w (2017).

[21] Wang, H.-W. et al. Forfeited hepatogenesis program and increased embryonic stem cell traits in young hepatocellular carcinoma (HCC) comparing to elderly HCC. BMC Genomics 14, doi: 10.1186/1471-2164-14-736. (2013).

[22] Chaisaingmongkol, J. et al. Common Molecular Subtypes Among Asian Hepatocellular Carcinoma and Cholangiocarcinoma. Cancer Cell 32, 57-70 e53, doi: 10.1016/j.ccell.2017.05.009 (2017).

[23] Wang, Y.-H. et al. Plasmalemmal Vesicle Associated Protein (PLVAP) as a therapeutic target for treatment of hepatocellular carcinoma. BMC Cancer 14, doi: 10.1186/1471-2407-14-815 (2014). 
[24] Wang, S. M., Ooi, L. L. \& Hui, K. M. Identification and validation of a novel gene signature associated with the recurrence of human hepatocellular carcinoma. Clin Cancer Res 13, 6275-6283, doi: 10.1158/1078-0432. CCR-06-2236 (2007).

[25] Chen, Y. L., Wang, T. H., Hsu, H. C., Yuan, R. H. \& Jeng, Y. M. Overexpression of CTHRC1 in hepatocellular carcinoma promotes tumor invasion and predicts poor prognosis. PLoS One 8, e70324, doi: 10.1371/journal.pone.0070324 (2013).

[26] $\mathrm{Li}, \mathrm{T}$. et al. TIMER2.0 for analysis of tumor-infiltrating immune cells. Nucleic Acids Res 48, W509-W514, doi: 10.1093/nar/gkaa407 (2020).

[27] Szklarczyk, D. et al. STRING v11: protein-protein association networks with increased coverage, supporting functional discovery in genome-wide experimental datasets. Nucleic Acids Res 47, D607-D613, doi: 10.1093/nar/gky1131 (2019).

[28] Zhou, Y. et al. Metascape provides a biologist-oriented resource for the analysis of systems-level datasets. Nat Commun 10, 1523, doi: 10.1038/s41467-019-09234-6 (2019).

[29] Azimi, F. et al. Tumor-infiltrating lymphocyte grade is an independent predictor of sentinel lymph node status and survival in patients with cutaneous melanoma. J Clin Oncol 30, 2678-2683, doi: 10.1200/JCO.2011.37.8539 (2012).

[30] Guo, S. \& Deng, C. X. Effect of Stromal Cells in Tumor Microenvironment on Metastasis Initiation. Int J Biol Sci 14 , 2083-2093, doi: 10.7150/ijbs.25720 (2018).

[31] Hinshaw, D. C. \& Shevde, L. A. The Tumor Microenvironment Innately Modulates Cancer Progression. Cancer Res 79, 4557-4566, doi: 10.1158/0008-5472. CAN-18-3962 (2019).

[32] Wu, T. \& Dai, Y. Tumor microenvironment and therapeutic response. Cancer Lett 387, 61-68, doi: 10.1016/j.canlet.2016.01.043 (2017).

[33] Sawant, A. et al. Depletion of plasmacytoid dendritic cells inhibits tumor growth and prevents bone metastasis of breast cancer cells. J Immunol 189, 4258-4265, doi: 10.4049/jimmunol.1101855 (2012).

[34] Zgajnar, N. R. et al. Biological Actions of the Hsp90-binding Immunophilins FKBP51 and FKBP52. Biomolecules 9, doi: 10.3390/biom9020052 (2019).

[35] Annett, S., Moore, G. \& Robson, T. FK506 binding proteins and inflammation related signalling pathways; basic biology, current status and future prospects for pharmacological intervention. Pharmacol Ther 215, 107623, doi: 10.1016/j.pharmthera.2020.107623 (2020).

[36] Alsaab, H. O. et al. PD-1 and PD-L1 Checkpoint Signaling Inhibition for Cancer Immunotherapy: Mechanism, Combinations, and Clinical Outcome. Front Pharmacol 8, 561, doi: 10.3389/fphar.2017.00561 (2017).

[37] Yakkundi, A. et al. FKBPL is a critical antiangiogenic regulator of developmental and pathological angiogenesis. Arterioscler Thromb Vasc Biol 35, 845-854, doi: 10.1161/ATVBAHA.114.304539 (2015).

[38] Liu, Y. et al. Proteomic mining in the dysplastic liver of WHV/c-myc mice--insights and indicators for early hepatocarcinogenesis. FEBS $J$ 277, 4039-4053, doi: 10.1111/j.1742-4658.2010.07795.x (2010).

[39] Lin, I.-Y. et al. Identification of FKBP11 as a biomarker for hepatocellular carcinoma. Anticancer Research 33, 2763-2769 (2013).

[40] Higgins, J. P. T. et al. Expression of FKBP12 in Benign and Malignant Vascular Endothelium: an Immunohistochemical Study on Conventional Sections and Tissue Microarrays. The American Journal of Surgical Pathology 27, 58-64, doi: 10.1097/00000478-200301000-00007. (2003).

[41] Khatua, S. et al. Overexpression of the EGFR/FKBP12/HIF-2 $\alpha$ Pathway Identified in Childhood Astrocytomas by Angiogenesis Gene Profiling. Cancer Research 63, 1865-1870 (2003).

[42] Erdmann, F., Jarczowski, F., Weiwad, M., Fischer, G. \& Edlich, F. Hsp90-mediated inhibition of FKBP38 regulates apoptosis in neuroblastoma cells. FEBS Lett 581, 5709-5714, doi: 10.1016/j.febslet.2007.11.037 (2007).

[43] Desmetz, C. et al. Identification of a new panel of serum autoantibodies associated with the presence of in situ carcinoma of the breast in younger women. Clin Cancer Res 15, 4733-4741, doi: 10.1158/1078-0432. CCR-08-3307 (2009).

[44] Lin, J. F. et al. Identification of candidate prostate cancer biomarkers in prostate needle biopsy specimens using proteomic analysis. Int $J$ Cancer 121, 2596-2605, doi: 10.1002/ijc.23016 (2007).

[45] Jiang, W. et al. FK506 binding protein mediates glioma cell growth and sensitivity to rapamycin treatment by regulating NF-kappaB signaling pathway. Neoplasia 10, 235-243, doi: 10.1593/neo.07929 (2008).

[46] Periyasamy, S., Hinds, T., Jr., Shemshedini, L., Shou, W. \& Sanchez, E. R. FKBP51 and Cyp40 are positive regulators of androgen-dependent prostate cancer cell growth and the targets of FK506 and cyclosporin A. Oncogene 29, 1691-1701, doi: 10.1038/onc.2009.458 (2010).

[47] Baughman, G., Wiederrecht, G. J., Campbell, N. F. \& Bourgeois, S. FKBP51, a Novel T-Cell-Specific Immunophilin Capable of Calcineurin Inhibition. Molecular and Cellular Biology 15, 4395-4402, doi: 10.1128/mcb.15.8.4395 (1995).

[48] Pei, H. et al. FKBP51 affects cancer cell response to chemotherapy by negatively regulating Akt. Cancer Cell 16, 259-266, doi: 10.1016/j.ccr.2009.07.016 (2009).

[49] Olesen, S. H. et al. Human FK506 binding protein 65 is associated with colorectal cancer. Mol Cell Proteomics 4, 534-544, doi: 10.1074/mcp. M400217-MCP200 (2005). 